\title{
HARMONIC DECOMPOSITION BY HHT ON REGULAR WAVES PROPAGATING OVER A RECTANGULAR SUBMERGED OBSTACLE UPON A FLUIDIZED BED
}

\author{
Yung-Lung Chen \\ Department of Harbor and River Engineering, National Taiwan Ocean University, Keelung, Taiwan, R.O.C., \\ d92520007@mail.ntou.edu.tw \\ Shiaw-Yih Tzang \\ Department of Harbor and River Engineering, National Taiwan Ocean University, Keelung, Taiwan, R.O.C. \\ Shan-Hwei Ou \\ Department of Environmental Resources Management, Tajen University, Pingtung, Taiwan, R.O.C.
}

Follow this and additional works at: https://jmstt.ntou.edu.tw/journal

Part of the Engineering Commons

\footnotetext{
Recommended Citation

Chen, Yung-Lung; Tzang, Shiaw-Yih; and Ou, Shan-Hwei (2011) "HARMONIC DECOMPOSITION BY HHT ON REGULAR WAVES PROPAGATING OVER A RECTANGULAR SUBMERGED OBSTACLE UPON A FLUIDIZED BED," Journal of Marine Science and Technology: Vol. 19: Iss. 6, Article 5.

DOI: $10.51400 / 2709-6998.2203$

Available at: https://jmstt.ntou.edu.tw/journal/vol19/iss6/5

This Research Article is brought to you for free and open access by Journal of Marine Science and Technology. It has been accepted for inclusion in Journal of Marine Science and Technology by an authorized editor of Journal of Marine Science and Technology.
} 
HARMONIC DECOMPOSITION BY HHT ON REGULAR WAVES PROPAGATING OVER A RECTANGULAR SUBMERGED OBSTACLE UPON A FLUIDIZED BED

Acknowledgements

This study is financially supported by the National Science Council, Taiwan, Republic of China under project numbers of NSC 97-2221-E-019-042. 


\title{
HARMONIC DECOMPOSITION BY HHT ON REGULAR WAVES PROPAGATING OVER A RECTANGULAR SUBMERGED OBSTACLE UPON A FLUIDIZED BED
}

\author{
Yung-Lung Chen*, Shiaw-Yih Tzang*, and Shan-Hwei Ou**
}

Key words: harmonic, wave decay, HHT, submerged obstacle, fluidized bed.

\begin{abstract}
The experimental measurements of surface waves over a submerged rectangular obstacle upon a sandy bed reported by Tzang et al. (2008) have further been analyzed in detail by Hilbert-Huang transform (HHT). The harmonic components of regular waves at 5 sites in the vicinity of the obstacle are decomposed into the intrinsic mode functions (IMFs) by the ensemble empirical mode decomposition (EEMD) with postprocessing scheme using masking signal EMD (MSEMD). The results of Hilbert spectrum of waves derived by the HHT illustrate that considerable decay of harmonic amplitude occurs at all 5 sites when the sandy bed have been fluidized but is not observed in any of the unfluidized tests. Much more reductions of amplitude due to the fluidization response are found in the fundamental harmonic particularly during the building-up of excess pore pressure than in higher harmonics.
\end{abstract}

\section{INTRODUCTION}

Wave flume experiments have been previously conducted to investigate wave reflection and decay of non-breaking regular waves propagating over a rectangular submerged obstacle (S.O.) upon a fluidized bed [10]. The results have illustrated that overpassing waves have significantly decayed after the underlying beds being fluidized. It is particularly found to be closely dependent on the development of excess pore pressure. The decayed waves could have become as small as half of those before the fluidization response.

In the past decades, many laboratory and numerical ex-

Paper submitted 02/25/10; revised 10/01/10, 02/12/11; accepted 03/14/11. Author for correspondence: Yung-Lung Chen (e-mail: d92520007@mail.ntou.edu.tw). *Department of Harbor and River Engineering, National Taiwan Ocean University, Keelung, Taiwan, R.O.C.

**Department of Environmental Resources Management, Tajen University, Pingtung, Taiwan, R.O.C. periments have been carried out to investigate waves propagating over impermeable or permeable submerged breakwaters. For example, Massel [7] presented a second order theory for waves passing over a step. Their results showed that the energy of first harmonic could be transferred to higher harmonics during passage over a structure, which are then further transmitted into free waves at the same frequency. Losada et al. [5] have shown the same behavior on a submerged porous step. They also found that waves with a lower Ursell number would become more unlikely to generate higher harmonics. Ohyama and Nadaoka [8] pointed out two-stage wave decomposition phenomenon during passage over a shelf. In the first stage there is the higher harmonic generation over the shelf. In the second stage there is a large amount wave energy transferring into free higher harmonics in the leeward of shelf.

According to soil mechanics, the fluidization responses occur when the pore pressures build up to the in-situ theoretical soil's static stresses. Tzang and $\mathrm{Ou}$ [9] had reported two types of wave-induced fluidized responses from their experiments on fine sandy beds including the resonant and nonresonant fluidization. Their resonant fluidization responses consist of significant build-ups in pore pressure and generally occur after the initiation of sudden amplification in the pore pressure oscillation. Since the fluidized pore pressure data are quite nonlinear and nonstationary, all Fourier based analysis techniques are incapable of analyzing these processes. Thus, the Hilbert-Huang transform (HHT) were selected and first validated in analyzing the nonstationary pore pressure data in a fluidization response [1].

The HHT is the novel and powerful data analysis technique for nonlinear and nonstationary processes that has been developed by Huang et al. [4]. The HHT consists of two main steps, the empirical mode decomposition (EMD) and Hilbert spectral analysis (HSA). The original EMD has a drawback on frequent appearance of mode mixing, hence the ensemble EMD (EEMD) is purposed by $\mathrm{Wu}$ and Huang [11] to alleviate mode mixing problem. However, still sometimes, the mode mixing problem can't be resolved completely by the EEMD. Thus, Chen et al. [2] demonstrated the capabilities and limitations of the EEMD with post processing using masking 
signal EMD (MSEMD) [3] on correcting the mode-mixed intrinsic mode function (IMF).

The interactions among wave, obstacle and fluidized bed have been addressed on the local variations [10]. To further detail the transformations of the wave harmonics induced by these interactions, the HHT method $[4,11]$ is chosen as a more appropriate tool to analyze wave data reported by Tzang et al. [10] for both unfluidized (UF) and resonantly fluidized (RF) responses. The harmonic components of frequencyshifted waves in the vicinity of the obstacle were first decomposed by the EEMD with post processing into intrinsic mode functions (IMFs). Then, the Hilbert spectra were derived to illustrate the temporal variations of each harmonic.

\section{HILBERT-HUANG TRANSFORM}

\section{Ensemble EMD and Post-Processing Method}

\section{1) Ensemble EMD}

The EEMD consists of an ensemble of decompositions of data added with different white noise series through multiple applications of EMD [11]. The ensemble means are then taken from a number of corresponding IMFs. The added white noise will distribute over the entire time frequency space and provide a reference scale for the signals to collate in one IMF. The individual IMF is very noisy but the means of corresponding IMFs will eventually cancel out the added white noise each other. The principal procedures of the EEMD are summarized as follows:

(1) Add a white noise to the targeted data;

(2) Decompose the added-noise data into IMFs by the EMD;

(3) Repeat steps (1) and (2) with different white noise each time;

(4) Obtain the ensemble means of corresponding IMFs as the final result.

The added white noise must have sufficiently large amplitudes to perturb the original data. The effect of the added white noise, then, can be governed by the well-established statistical rule [11]:

$$
\varepsilon_{n}=\frac{\varepsilon}{\sqrt{N}}
$$

where $N$ is the number of ensemble members, $\varepsilon$ is the amplitude of the added noise, and $\varepsilon_{n}$ is the final standard deviation of error, which is defined as the difference between the input signal and the corresponding IMFs. About the EMD sifting stopping criterion, in this study we adopted two conditions, including (i) the standard deviation $S D$ [4] is lower than a small value (i.e. $10^{-3}$ ) and (ii) the number of extrema and zero crossings $D N$ differ at most by one.

2) Post-Processing Method Using EMD
The IMFs derived from the EEMD are not real IMFs owing to the ensemble average of the different sets of IMFs. These IMFs are not suitable for Hilbert spectral analysis and do not satisfy the IMF criteria as shown below:

(i) The number of extrema and the number of zero crossings must either equal or differ at most by one.

(ii) At any point, the local mean is zero.

To overcome these problems, $\mathrm{Wu}$ and Huang [11] introduced a post-processing method using EMD. The detailed algorithm of this post-processing can be referred to $\mathrm{Wu}$ and Huang [11]. Since the mode mixing is in general often caused by high-frequency intermittence, the EEMD-processed components are demanded for additional EMD calculation.

\section{3) Post-Processing Method Using MSEMD}

The EEMD-processed data sometimes still have the drawback of mode mixing. Here, another post-processing method proposed by Chen et al. [2] is applied to modify the modemixed IMF. They have reported that through the MSEMD the mode-mixed EEMD IMF could be separated well. The modified EEMD procedure with the MSEMD as a post-processing method is shown in Fig. 1. The main concept is to insert a masking signal $S(t)$, a simple sine wave whose frequency being close to higher-frequency component, to the mode-mixed IMF $C_{i}$ and then perform EMD to extract higher-frequency component. Once an IMF is modified, an additional post processing using EMD would further be operated on the IMFs. The detailed description of the post processing method using MSEMD can be referred to Chen et al. [2].

\section{Hilbert Spectral Analysis}

Once the intrinsic mode function components are obtained, the Hilbert spectral analysis is applied to represent timefrequency-distribution of the amplitude. The Hilbert transform is performed on each $\operatorname{IMF} C_{j}(t)$; it can be expressed as follows:

$$
\tilde{C}_{j}(t)=\frac{1}{\pi} P V \int_{-\infty}^{\infty} \frac{C_{j}\left(t^{\prime}\right)}{t-t^{\prime}} d t^{\prime}
$$

where $P V$ indicates the Cauchy principal value of singular integral. Essentially, Eq. (2) defines the Hilbert transform as the convolution of $C_{j}(t)$ with $1 / \pi t$. With the Hilbert transform, the analytic signal $X(t)$ is defined as

$$
X(t)=C_{j}(t)+i \tilde{C}_{j}(t)=a_{j}(t) e^{i \theta_{j}(t)}
$$

in which

$$
\begin{gathered}
a_{j}(t)=\left[C_{j}^{2}(t)+\tilde{C}_{j}^{2}(t)\right]^{1 / 2} \\
\theta_{j}(t)=\tan ^{-1}\left(\frac{\tilde{C}_{j}(t)}{C_{j}(t)}\right)
\end{gathered}
$$




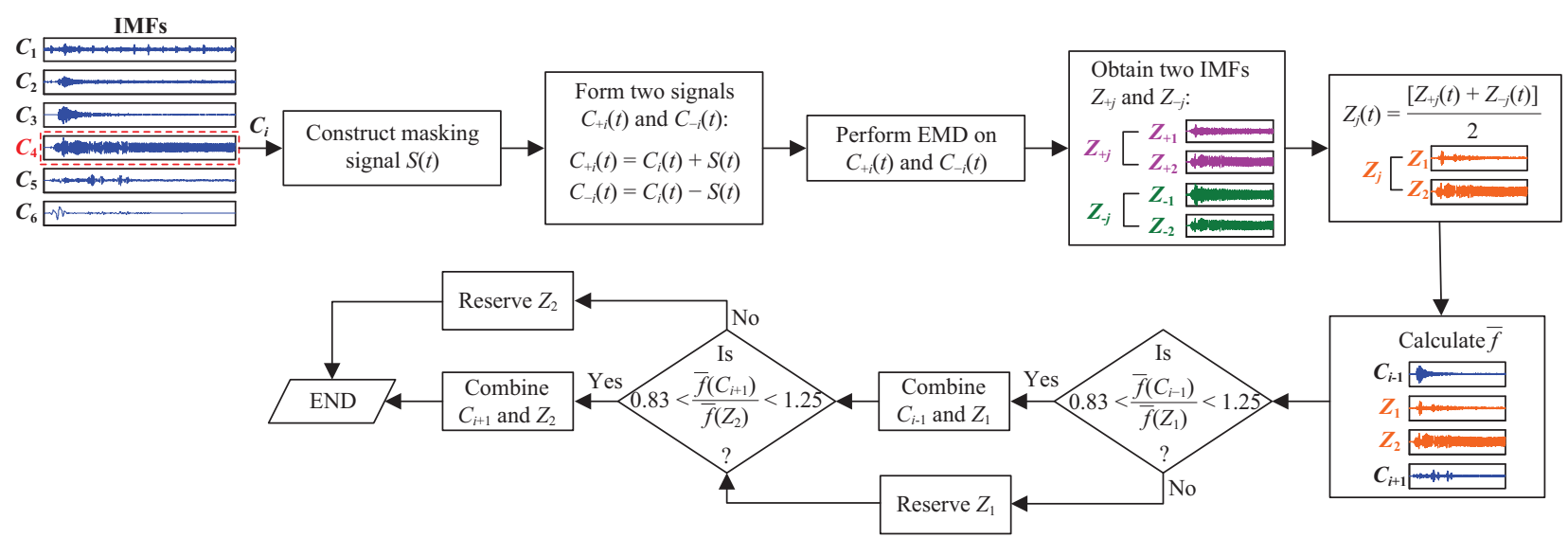

Fig. 1. Flowchart of EEMD post-processing method using MSEMD.

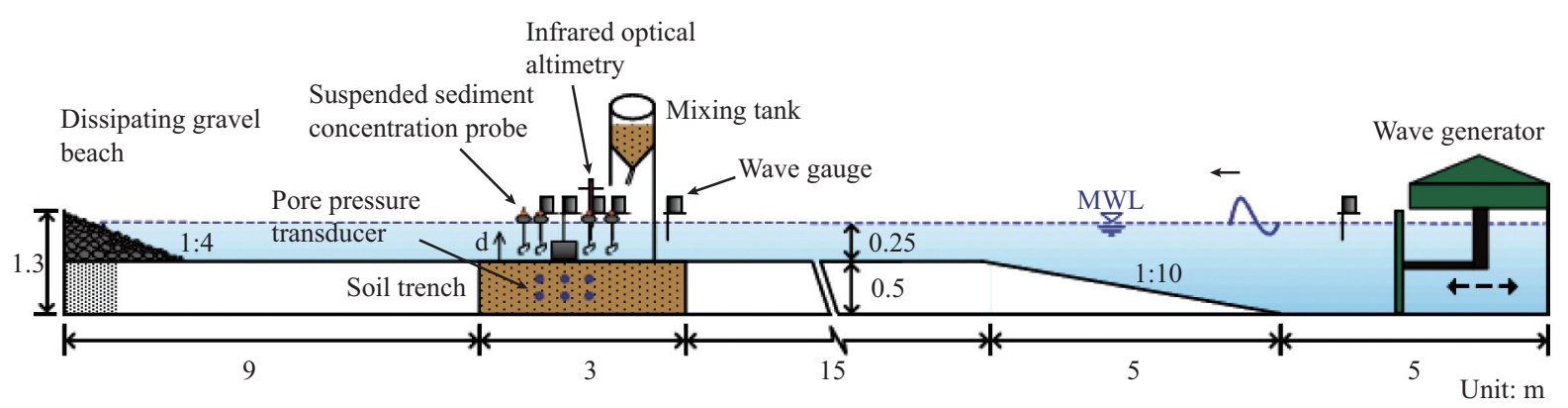

Fig. 2. Sketch of wave flume set-ups (unit: $\mathrm{m}$ ).

The $a_{j}(t)$ is the instantaneous amplitude, and the $\theta_{j}(t)$ is the instantaneous phase. The IMF admits well-behaved Hilbert transforms and the instantaneous frequency (IF) is derived by differentiation of the instantaneous phase function as:

$$
f_{j}(t)=\frac{1}{2 \pi} \frac{d \theta_{j}(t)}{d t}
$$

The time-frequency-distribution of the amplitude is designated as the Hilbert amplitude spectrum $H S(f, t)$. Thus, the marginal spectrum $M S(f)$ can be derived as:

$$
M S(f)=\frac{1}{T_{D}} \int_{0}^{T_{D}} H S(f, t) d t
$$

where $T_{D}$ is the total data length.

\section{WAVE FLUME MEASUREMENTS}

\section{Experimental Set-ups and Test Conditions}

As shown in Fig. 2, the selected experiments were conducted in a $37 \mathrm{~m}$ (length) $\times 1 \mathrm{~m}$ (width) $\times 1.2 \mathrm{~m}$ (height) wave flume with an indented soil trench, $3 \mathrm{~m}$ (length) $\times 1 \mathrm{~m}$ (width) $\times 0.5 \mathrm{~m}$ (height). As shown in Fig. 3, the soil trench was filled with a fine sand $\left(d_{50}=0.073 \mathrm{~mm}\right)$ at a constant water

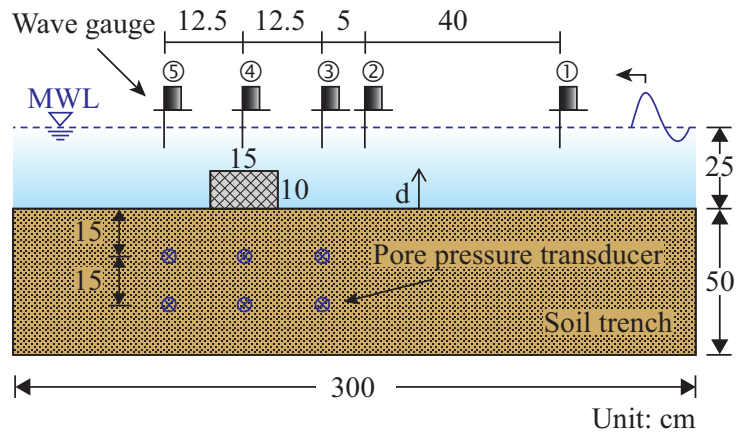

Fig. 3. Experimental set-ups (unit: cm).

depth of $25 \mathrm{~cm}$. The impermeably submerged obstacle (S.O.) was made of transparent acrylic box with a height of $10 \mathrm{~cm}$ and a thickness of $15 \mathrm{~cm}$ with the same width of the wave flume.

The wave measurements reported by Tzang et al. [10] were analyzed here in detail by applying the HHT. Five wave gauges were installed above the soil trench with one above the obstacle, one at location of $5 \mathrm{~cm}$ downstream of the obstacle, and three at locations of $5 \mathrm{~cm}, 10 \mathrm{~cm}$ and $50 \mathrm{~cm}$ upstream of the obstacle. Six pore pressure transducers inside the bed were installed in three vertical sections at two depths, i.e. $15 \mathrm{~cm}$ and $30 \mathrm{~cm}$ below bed surface $(d=-15 \mathrm{~cm}$ and $-30 \mathrm{~cm})$. Each test 
Table 1. Summary of design test conditions and typical results.

\begin{tabular}{|l|c|c|c|c|c|c|c|c|}
\hline Test & $H(\mathrm{~cm})$ & $T(\mathrm{~s})$ & $H / L$ & $h / L$ & $U_{r}$ & $K_{r}$ & Response & $d_{f}(\mathrm{~cm})$ \\
\hline A-5 & 5.86 & 1.1 & 0.040 & 0.169 & 8.25 & 0.14 & UF & 1.8 \\
\hline A-6 & 6.32 & 1.1 & 0.043 & 0.169 & 8.90 & 0.15 & RF & 18.9 \\
\hline B-3 & 4.81 & 1.5 & 0.022 & 0.115 & 14.54 & 0.14 & UF & 1.6 \\
\hline B-4 & 4.93 & 1.5 & 0.023 & 0.115 & 14.90 & 0.13 & RF & 23.7 \\
\hline C-2 & 4.13 & 2.0 & 0.014 & 0.083 & 23.79 & 0.14 & UF & 2.3 \\
\hline C-4 & 6.03 & 2.0 & 0.020 & 0.083 & 34.74 & 0.17 & RF & 25.9 \\
\hline$H:$ wave height & \multicolumn{1}{|c|}{$K_{r}:$ reflection coefficient } \\
$T:$ wave period & $d_{f}:$ thickness of fluidized soil-layer \\
$L:$ wavelength & UF: unfluidized \\
$H:$ water depth \\
$U_{r}:$ Ursell number
\end{tabular}

series consists of at least 9 tests and the test conditions are summarized in Table 1 for further analysis. Each test series stands for conducting waves from on a freshly deposited soil to on pre-loaded one with consolidation periods of about 3 to 4 hours between two consecutive tests. Detailed information on the test soil bed preparation, test procedures and data acquisition can be referred to Tzang and $\mathrm{Ou}$ [9]. In this study, only 2 tests of each series are listed here with one UF test and one RF test. The Ursell number is defined as $U_{r}=H L^{2} / h^{3}$, in which $h$ is the water depth, and $H$ and $L$ are wave height and wavelength. The wave reflection coefficient $K_{r}$ is calculated by applying the scheme, based on spectrum analysis by Mansard and Funke [6], to records of the three consecutive wave gauges No. 1 3 upstream of the S.O.

\section{Wave and Pore Pressure Records}

The fluidized response is herein defined as a process that some portions of the soil's solid skeleton lose their intergranular contacts and are born by pore fluid resulting in compensated pore pressure build-ups (or excess pore pressure) [9]. Hence, in this study, the corresponding thickness of fluidized soil-layer, $d_{f}$, is derived from the maximum excess pore pressure $\left(\Delta P_{\max }\right)$ by:

$$
\Delta P_{\max }=(1-n)\left(\rho_{s}-\rho_{w}\right) g d_{f}
$$

where $\rho_{s}$ and $\rho_{w}$ are the densities of soil's solid particles and pore water of 2640 and $1000 \mathrm{~kg} / \mathrm{m}^{3}, n$ is the porosity $(0.48)$ and $g$ is the gravitational acceleration $\left(9.81 \mathrm{~m} / \mathrm{s}^{2}\right)$. At a depth of $30 \mathrm{~cm}(d=-30 \mathrm{~cm})$ inside the bed, the upper limit of the excess pore pressure $\Delta P_{s}$ is theoretically equal to $2510 \mathrm{~N} / \mathrm{m}^{2}$ for the present sandy soil.

Fig. 4 displays the typical simultaneous measurements of surface waves and pore pressures from the UF Test B-3. The wave records were measured from wave gauges No. 3 , No. 4 and No. 5, and pore pressure records were measured at a depth of $30 \mathrm{~cm}(d=-30 \mathrm{~cm})$ below the bed surface along the same horizontal sites as wave gauges of No. 3 5. The wave gauges (a)
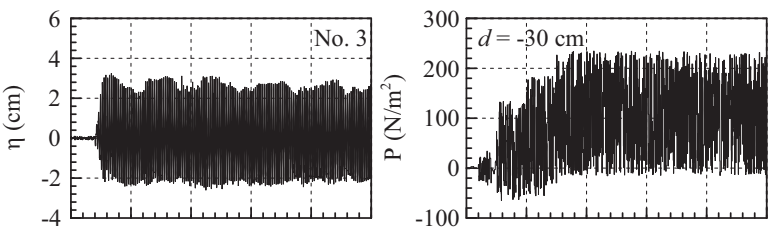

(b)
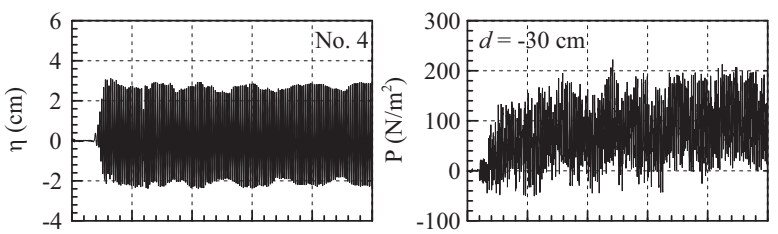

(c)
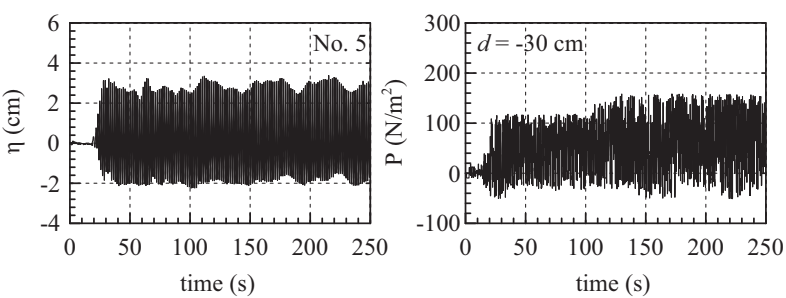

Fig. 4. Synchronous records of wave and pore pressure in a depth of $\mathbf{3 0}$ cm along wave propagation from UF Test B-3 at locations of (a) 5 cm upstream (No. 3), (b) midpoint (No. 4), and (c) 5 cm downstream (No. 5) of the S.O.

(a)
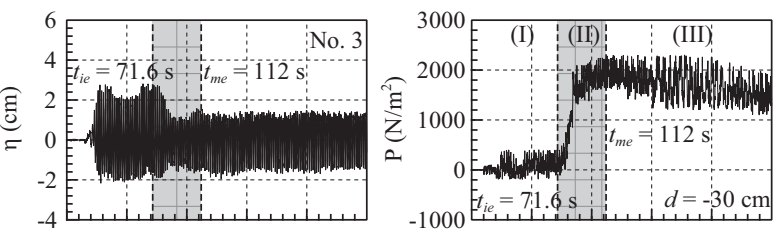

(b)
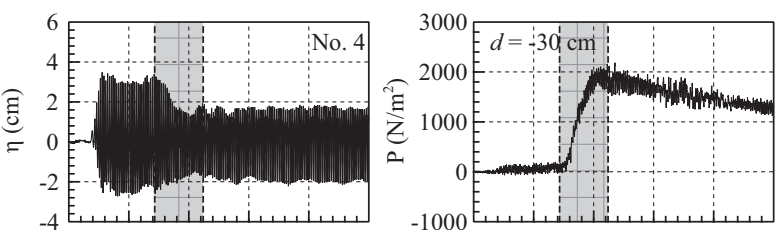

(c)
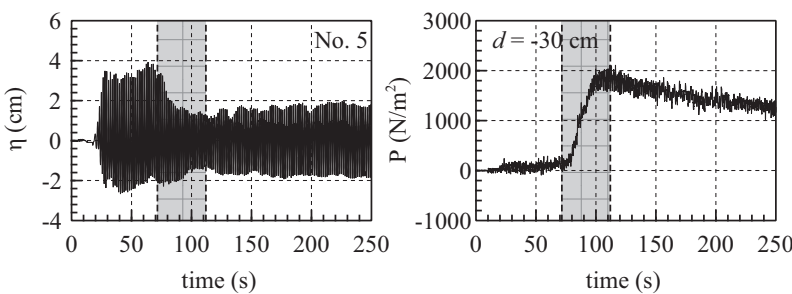

Fig. 5. Synchronous records of wave and pore pressure in a depth of 30 cm along wave propagation from RF Test B-4 at locations of (a) 5 cm upstream (No. 3), (b) midpoint (No. 4), and (c) $5 \mathrm{~cm}$ downstream (No. 5) of the S.O.

are located $5 \mathrm{~cm}$ upstream, midpoint, and $5 \mathrm{~cm}$ downstream of the S.O. It is seen in Fig. 4 that in the unfluidized bed waveinduced pore pressures have begun to build up after being subjected to loading waves and then attained to a relatively small steady value (e.g. $\left.131 \mathrm{~N} / \mathrm{m}^{2}\right)$. Meanwhile, the wave 
(a)

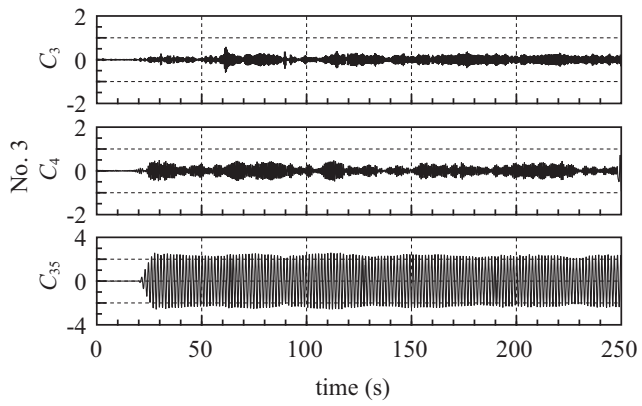

(b)

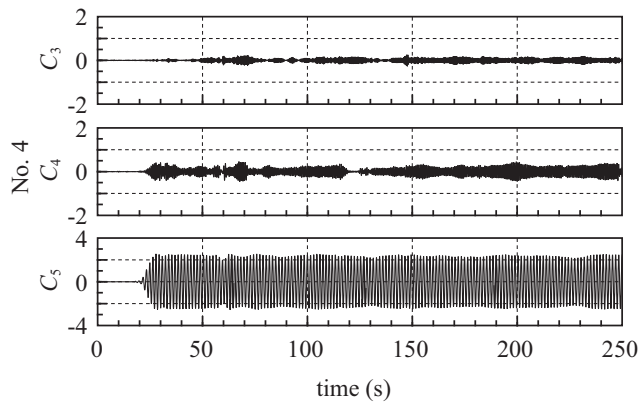

(c)

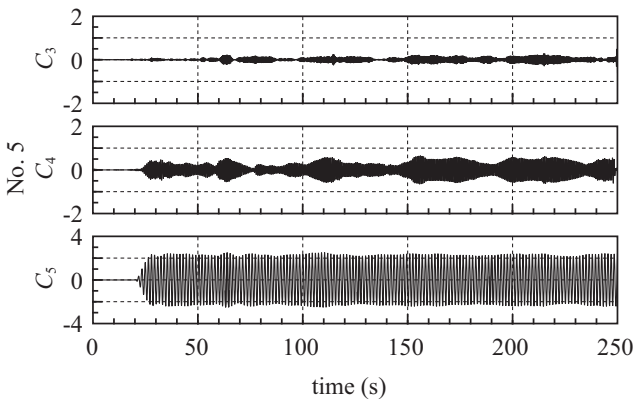

Fig. 6. IMFs of wave records of UF Test B-3 at locations of (a) $5 \mathrm{~cm}$ upstream (No. 3), (b) midpoint (No. 4), and (c) $5 \mathrm{~cm}$ downstream (No. 5) of the S.O.

records display quite similar patterns through the response at the three sites without any evident changes in the amplitudes of waves.

On the other hand, Fig. 5 demonstrates the typical measurements of surface waves and pore pressures from the RF Test B-4. Based on previous findings, the RF response should be the first test of fluidized response in a test series. Moreover, a RF test include a typical resonance mechanism being illustrated by sharp amplitude amplification within the fluidized layer. The pore pressures initially build up at time of $71.6 \mathrm{~s}\left(t_{i e}\right)$ and approach to a maximum value $\left(\Delta P_{\max }\right)$ of $1986 \mathrm{~N} / \mathrm{m}^{2}$ at time of $112 \mathrm{~s}\left(t_{m e}\right)$. The value of $\Delta P_{\max }$ accounts for about $79 \%$ of the theoretical soil stress $\left(2510 \mathrm{~N} / \mathrm{m}^{2}\right)$ at that depth, which also suggest that the measurements were not taken within the fluidized layer so that the resonant amplitude amplification are not evident in the records of Fig. 5. By the two defined times $t_{i e}$ and $t_{m e}$, the pore pressure variations of a fluidized response can be divided into three stages: (I) pre-fluidized, (II) transient and (III) post-fluidized stages. In transient stage, the shadowed zone in the figure, it can be clearly noted that the ampli- (a)

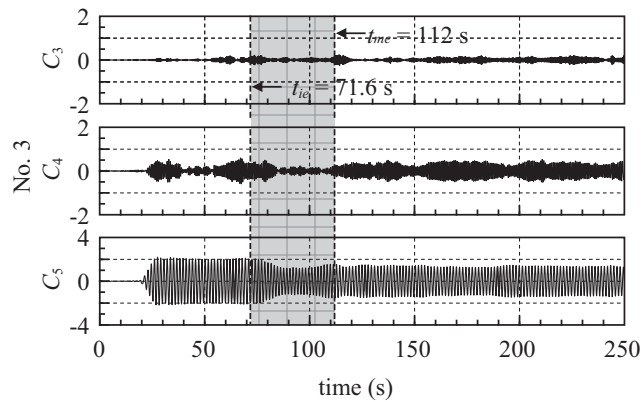

(b)

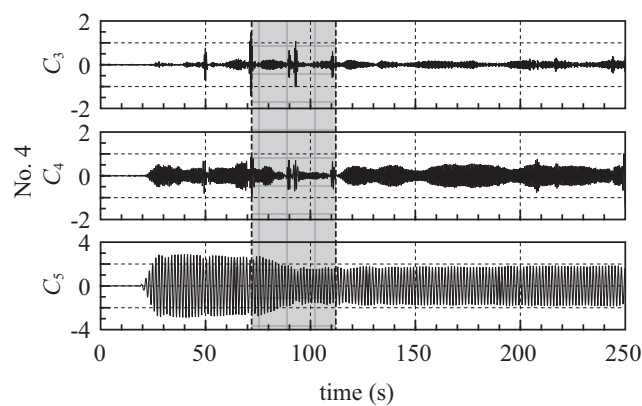

(c)

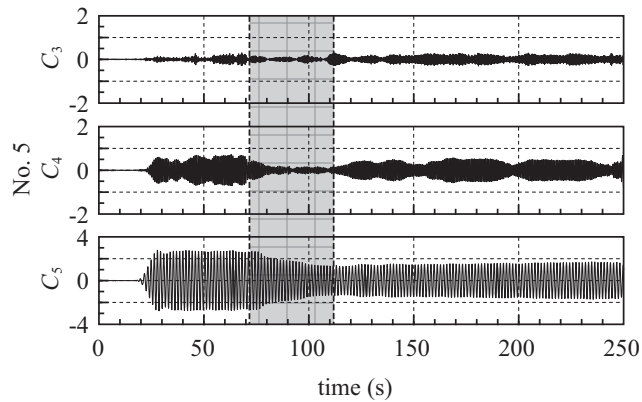

Fig. 7. IMFs of wave records of RF Test B-4 at locations of (a) 5 cm upstream (No. 3), (b) midpoint (No. 4), and (c) $5 \mathrm{~cm}$ downstream (No. 5) of the S.O.

tudes of waves begin to attenuate after excess pore pressures having increased. In post-fluidized stage as excess pore pressures have increased to a maximum value at $t_{m e}$, waves are seen to cease to decay.

\section{RESULTS AND DISSCUSSIONS}

\section{Wave Decomposition and Time-Frequency Distribution}

Wave measurements of UF and RF tests, as shown in Fig. 4 and Fig. 5 were then decomposed into IMFs by EEMD. Fig. 6 shows the IMFs of surface waves of the UF Test B-3 in Fig. 4 from wave gauges of No. 3 5. The IMFs displayed in following figures are only those components which carry the majority of the embedded energy in the records of surface wave. It is clearly noted that IMF $C_{5}$ has much larger amplitude than IMF $C_{3}$ and $C_{4}$. They coexist from the first wave indicating weak nonlinearity in the initial generation of the loading waves. In addition, the amplitude of IMF $C_{5}$ oscillates with nearly constant magnitude while those of IMF $C_{3}$ and $C_{4}$ oscillate with varying amplitudes. This suggests that higher- 

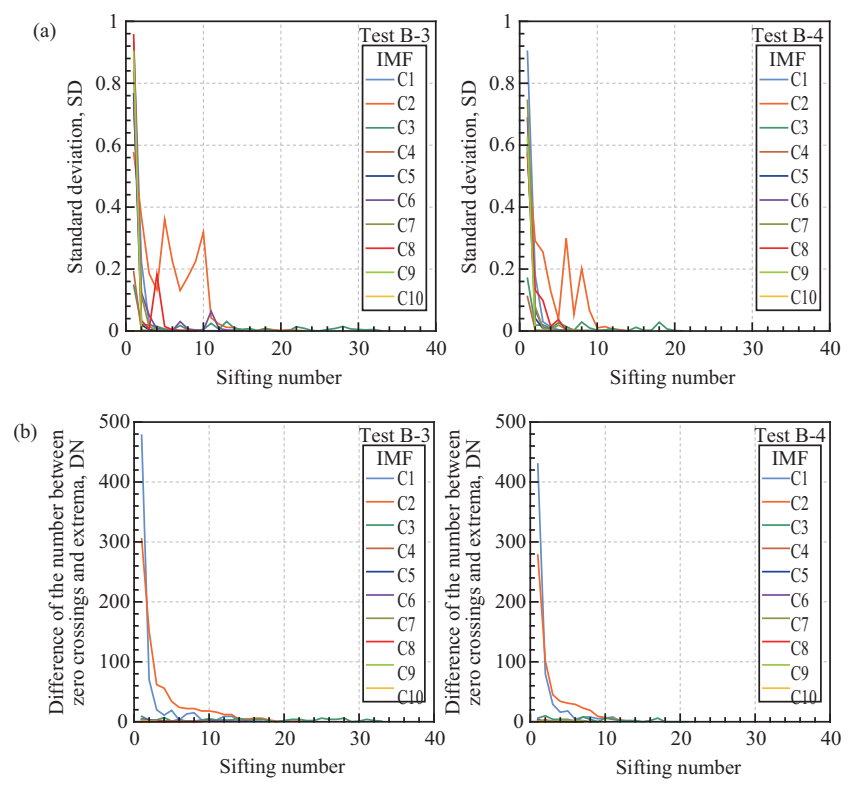

Fig. 8. (a) The standard deviation and (b) the difference of the number between zero crossings and extrema as a function of sifting numbers in UF Test B-3 and RF Test B-4.

harmonic components are more susceptible to wave-submerged obstacle interactions.

Fig. 7 displays the IMFs of surface waves in Fig. 5 from RF Test B-4. In pre-fluidized stage $(t<71.6 \mathrm{~s})$, the amplitude variations of IMFs $C_{3} \sim C_{5}$ have similar trends as in UF Test B-3. The shadow zone in each subfigure indicates the transient stage (71.6 $\mathrm{s}<t<112 \mathrm{~s})$. In the transient stage, amplitudes of IMF $C_{4}$ and $C_{5}$ have evidently decayed during the build-up of excess pore pressures. After reaching maximum values after $t=112 \mathrm{~s}$, excess pore pressures in Fig. 5 are seen to decrease gradually while the amplitudes of IMF $C_{5}$ in Fig. 7 are noted to have slowly increased until the end of records. Especially, those of IMF $C_{4}$ have rapidly increased to similar magnitude in the pre-fluidized stage after $t=112 \mathrm{~s}$.

As mentioned above, in this study the number of iteration for EMD sifting process is selected according to two criteria. The first depends on the $S D$ values between two consecutively sifted components. As shown in Fig. 8(a), the $S D$ variations for each IMF from wave gauges No. 5 of the UF Test B-3 and the RF Test B-4 immediately illustrate that the $S D$ drops rapidly through only a few siftings. The second is judged from the $D N$ values. This is clearly seen in Fig. 8(b), in which the $D N$ values also decrease rapidly in the first two IMFs while the other IMFs have relatively slow decreases due to the lower values of initial $D N$. Moreover, it is noted that the maximum sifting numbers $N_{\max }$ are all smaller than a value of 50. To compare all the tests listed in Table 1, the detailed $N_{\max }$ values of the corresponding IMFs shown in Fig. 9 clearly indicate that the values of $N_{\max }$ of the corresponding IMFs in the six tests are all smaller than 50. Larger numbers of sifting are typically found in higher-frequency IMFs (i.e. the first four IMFs $C_{1} \sim C_{4}$ ).

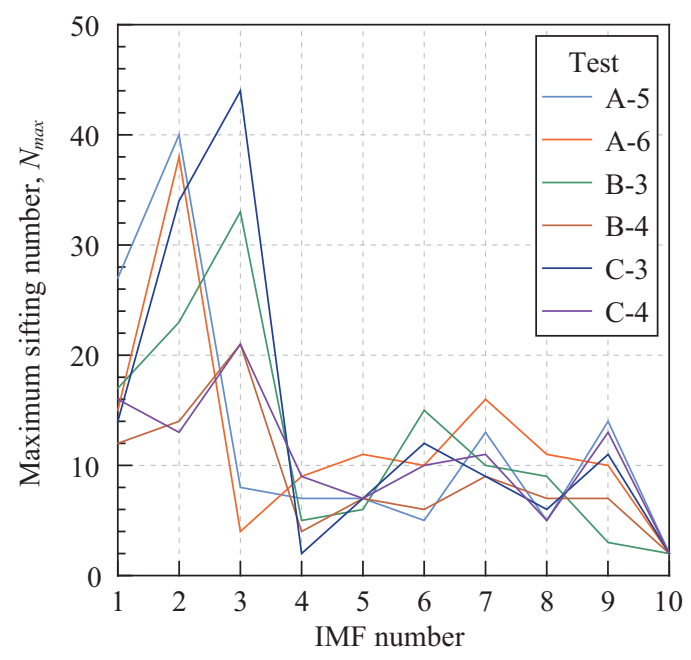

Fig. 9. The maximum sifting numbers of corresponding IMFs.

Fig. 10 further shows the Hilbert amplitude spectra of IMFs $C_{3} \sim C_{5}$ from wave gauges No. 1 5 for UF Test B-3. All panels are displayed with contour maps and the color-bars adjacent to right subfigures indicate amplitude magnitudes. For evident exhibition of time-frequency distribution, the ranges of the color bars for IMF $C_{3}$ and $C_{4}$ are redefined to be narrower than those for IMF $C_{5}$. In the bottom panel of each column, the time-dependent Hilbert spectra illustrate that IMF $C_{5}$ is the fundamental- or first-harmonic component with the range of the instantaneous frequency (IF) to be between $0.6 \mathrm{~Hz}$ and 0.7 Hz. The IMF $C_{4}$ and $C_{3}$ are identified to be the second and third harmonics with frequency ranges of $1.1 \mathrm{~Hz} \sim 1.5 \mathrm{~Hz}$ and $1.8 \mathrm{~Hz} \sim 2.4 \mathrm{~Hz}$, respectively. It is clear that wider frequency ranges are found to be associated with higher harmonics.

On the upstream side of S.O., measurements of wave gauge No. 3 show relatively larger amplitude of IMF $C_{3}$ and smaller amplitude of IMF $C_{4}$ than those of gauges No. 1 and No. 2. This includes slight growth of the third harmonic when the waves propagate close to the S.O. Furthermore, as waves propagate over the S.O. and leeward, wave records from gauges of No. 4 and No. 5 have illustrated the growth of higherfrequency components due to wave-obstacle interactions.

Fig. 11 illustrates the Hilbert amplitude spectra of IMFs $C_{3} \sim C_{5}$ from RF Test B-4. In the transient stage (71.6 $\mathrm{s}<t<$ $112 \mathrm{~s}$ ), considerable decay is observed in fundamental harmonic $C_{5}$. Meanwhile in the second and third harmonics are also found the significant decay and the wider frequency range. This simply indicates that the harmonic amplitudes rapidly decrease due to the continuous build-ups of pore pressure. It may be noted that, at gauge No. 1, the magnitude of the energy decay is less significant than the other four records (No. 2 5) suggesting relatively significant wave-amplitude decay in major harmonics of waves around the S.O. In post-fluidized stage, the amplitudes of fundamental harmonic $C_{5}$ increase gradually due to the drop of the excess pore pressure (as shown in Fig. 5). However, unlike the fundamental harmonic, 

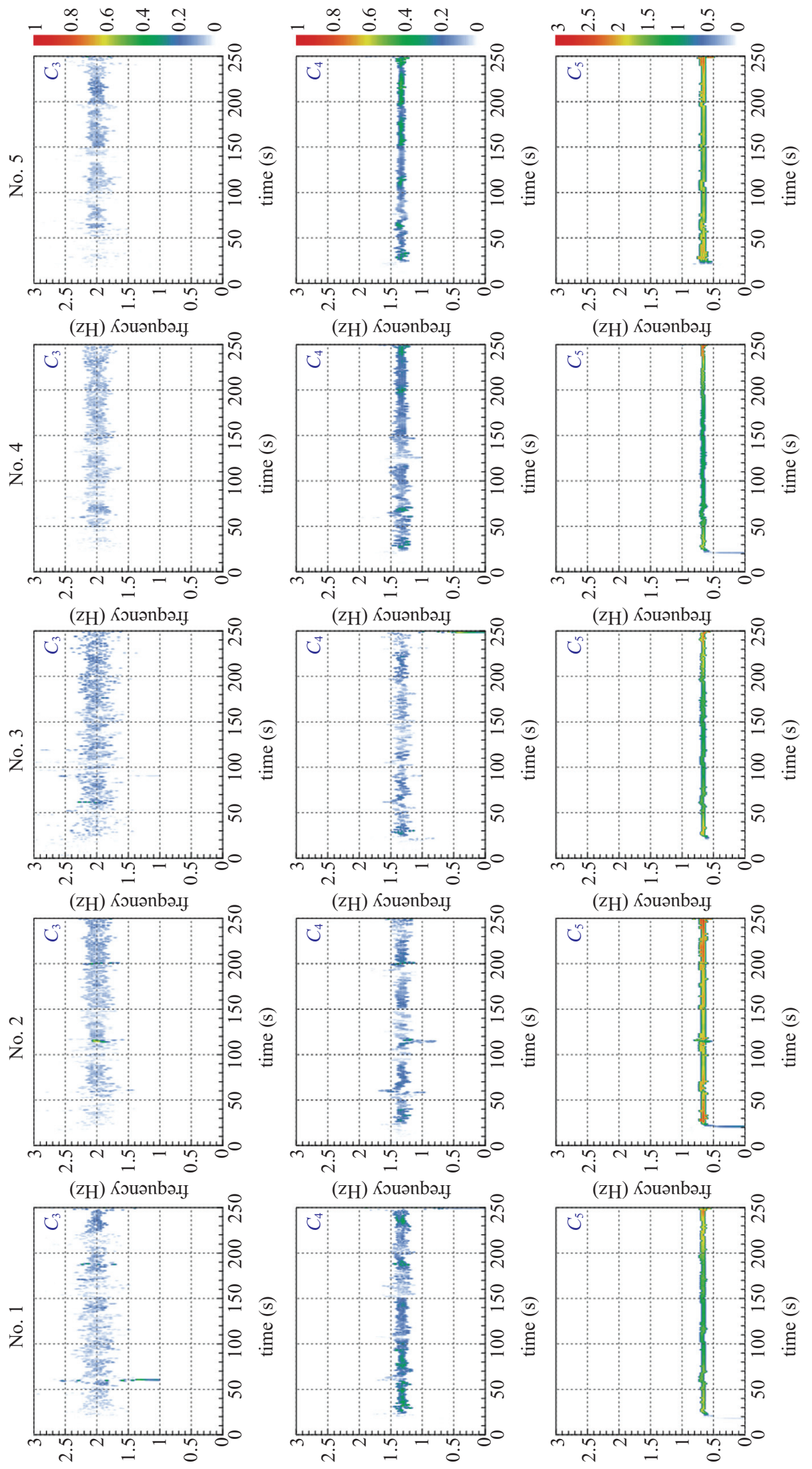

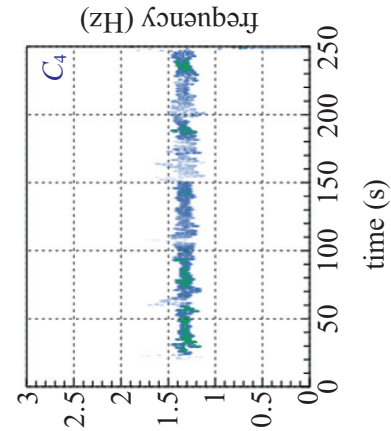

(zH) Kəuənbəry

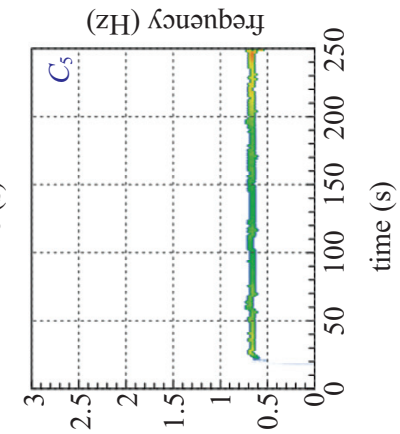

(zH) Kəuənbə.j

Fig. 10. Hilbert spectra of wave records along wave propagation from UF Test B-3. 

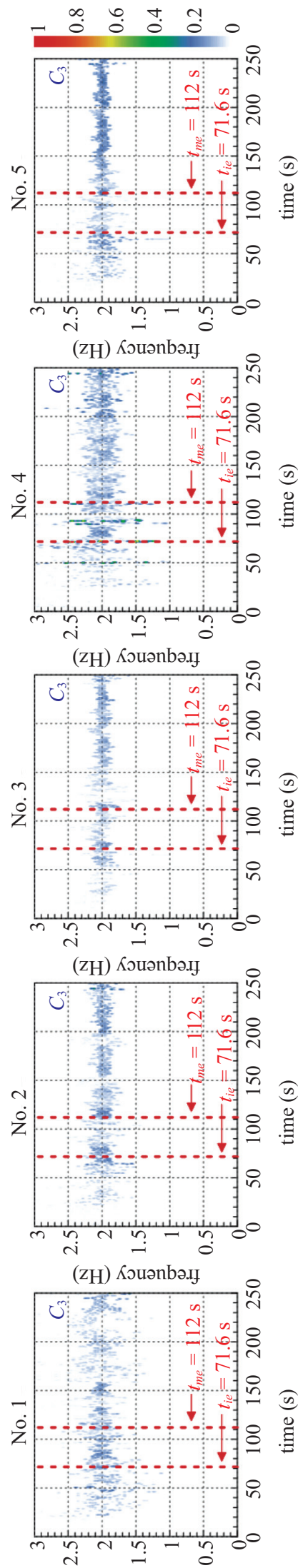

(zH) К๖uәnbə.I
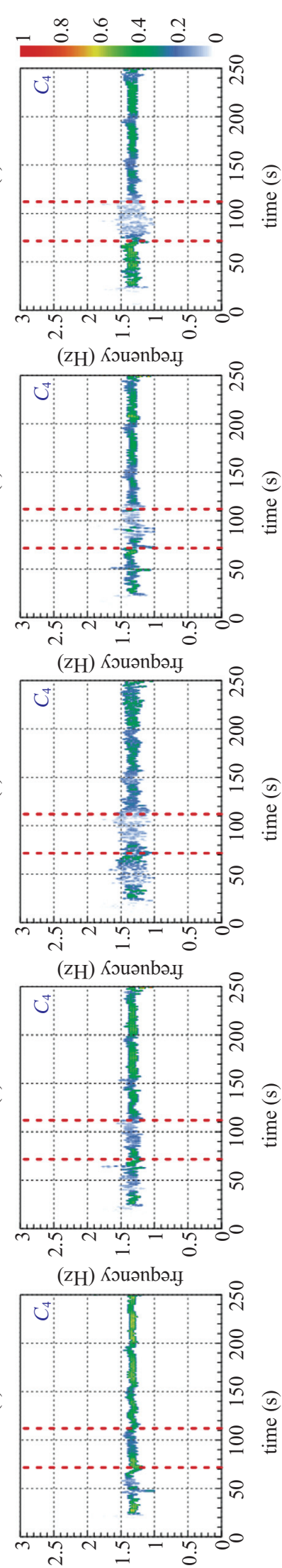

(zH) Kouənbə.I

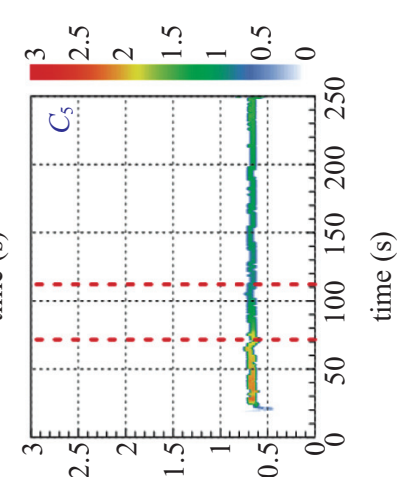

(zH) Kəuənbəry

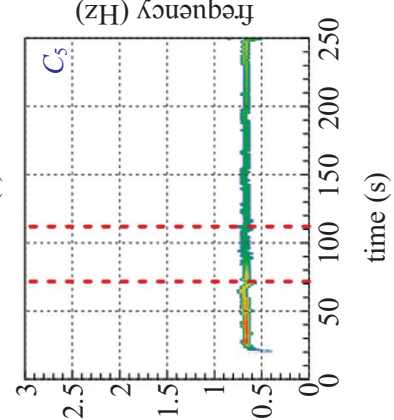

(zH) Kouənbəry

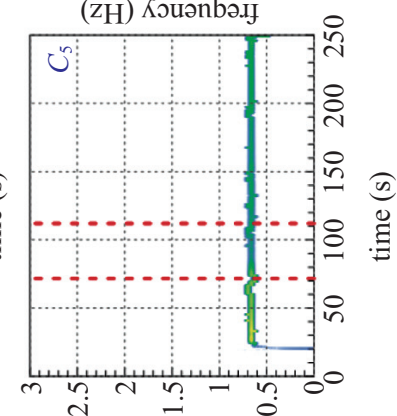

(zH) Кэuənbə.у

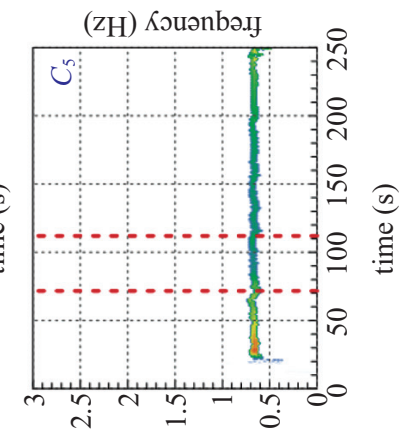

(zH) Kouənbəry

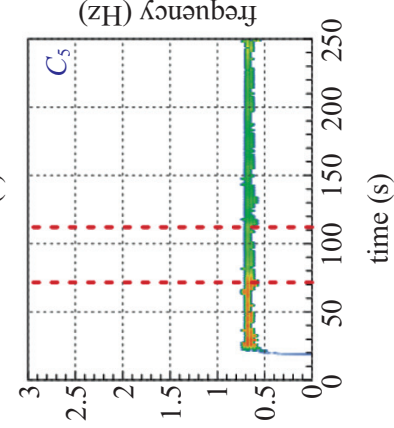

(zH) Kouənbə.J

Fig. 11. Hilbert spectra of wave records along wave propagation from RF Test B-4. 

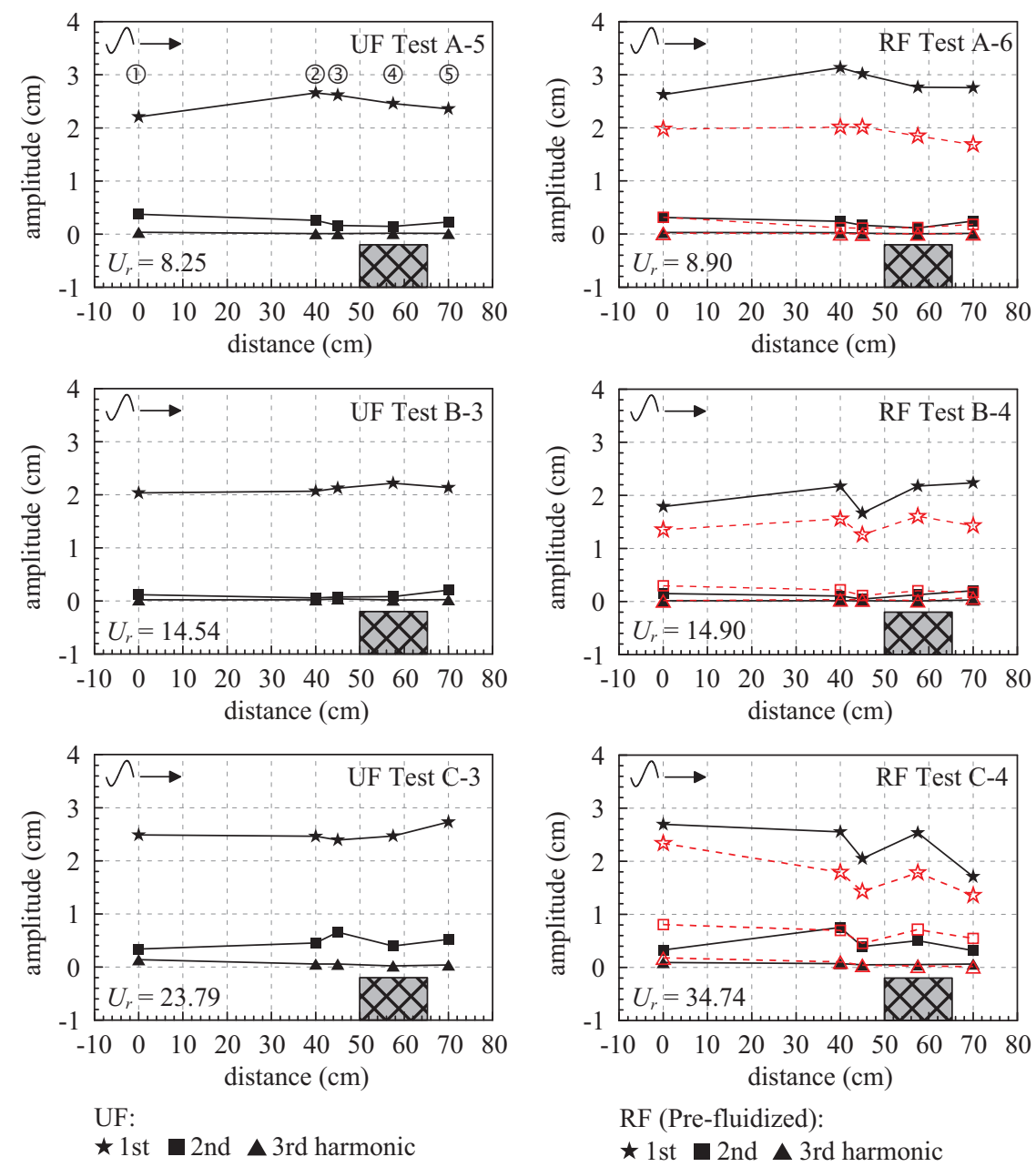

(a) UF tests

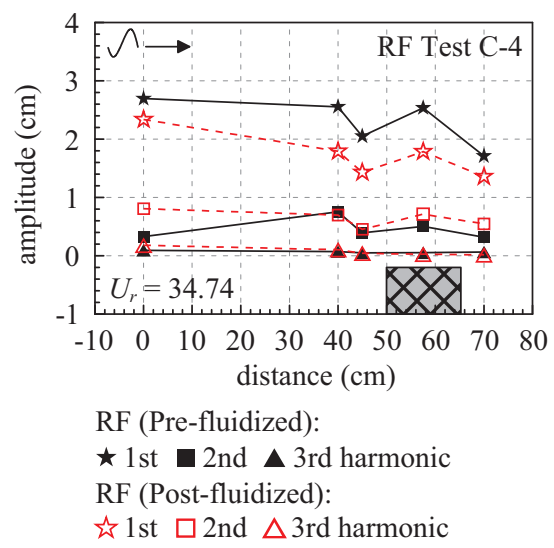

(b) RF tests

Fig. 12. Spatial evolution of the amplitude of first three harmonics from the five wave gauges in (a) UF and (b) RF tests (distance is defined as the downstream span from the first gauge).

the amplitudes of the higher harmonics $C_{3}$ and $C_{4}$ increase more rapidly after $t=112 \mathrm{~s}$ to the original magnitude in prefluidized stage.

\section{Comparison between UF and RF tests}

The spatial distributions of mean amplitudes of the three harmonics at gauges No. 1 5 for UF and RF tests are respectively shown in Fig. 12. The horizontal axes in Fig. 12 are the distance from wave gauge No. 1. The harmonic amplitudes are derived from the peak values of marginal spectrum (MS) being calculated from Eq. (7). For UF tests, the marginal spectra are estimated with whole recorded wave data. However, for RF tests, the wave records are divided into three parts to derive the marginal spectra based on the three stages of the build-ups of pore pressure (as shown in Fig. 5). Thus, in Fig. 12, RF tests have two values at each location; the filled symbols designate the marginal amplitudes in pre-fluidized stage, and the hollow symbols mark the amplitudes in post-fluidized stage.

As shown Fig. 12(a), as waves propagate over the S.O., the three UF tests do not reveal a similar dissipation pattern in the fundamental harmonic to that reported by Losada et al. [5]. Previous references indicate that the wave transmission above the S.O. is related to the relative freeboard and its relative width. The two dimensionless parameters are defined as $h_{s} / h$ and $D / h$ respectively, where $h_{s}$ is the depth of the submergence, $h$ is the water depth and $D$ is the width of S.O. The values of the two parameters in this study are $0.6\left(h_{s} / h\right)$ and $0.6(D / h)$, respectively. As for Losada et al. [5], the two corresponding parameters are $0.19\left(h_{s} / h\right)$ and $1.08(D / h)$, respectively. It is clear that the relative freeboard in this study is larger than that of Losada et al. while the relative width of S.O. is smaller than theirs. This indicates that for present study the effect of S.O. along on the growth of the nonlinear harmonic might not be notable. For Test C-3, the higher-harmonic amplitudes are 
relatively larger than those of other two tests possibly due to stronger initial nonlinearity $\left(U_{r}=32.55\right)$, as shown in Table 1 . However, as waves propagate over the S.O. upon an unfluidized sandy bed, the results reveal slightly larger amplitudes of higher harmonics on the leeward side of the S.O. than those above the S.O. That is, higher harmonics are likely mainly generated by the wave nonlinearity due to greater values of $U_{r}$ while the effects of the S.O. seem quite insignificant without the occurrence of a fluidization event.

In the top panel of Fig. 12(b), for RF Test A-6, the spatial distributions of the harmonic amplitude in pre-fluidized stage display similar results as those of UF Test A-5. However, results of RF Test B-4 or RF Test C-4 show different trends around the S.O. in pre-fluidized stage, even both the UF and RF tests have similar wave conditions. As shown in the middle and bottom panels of Fig. 12(b), for RF Test B-4 and Test $\mathrm{C}-4$, as waves arrive at the location of gauge No. 3 , the amplitudes decrease in fundamental and second harmonics. When waves further propagate over the S.O., the harmonic amplitudes are observed to increase. Moreover, on leeward side of the S.O., the decrease of the amplitude is only observed in Test C-4 while Test B-4 has nearly identical amplitudes as those over the S.O. No clear explanations so far are valid for different outcomes between UF and RF tests in pre-fluidized stage. But the results have suggested that the spatial evolutions of wave amplitudes with larger $U_{r}$ are significantly influenced by the S.O.

As shown in Fig. 12(b), in post-fluidized stage, the firstharmonic amplitudes have shown considerable reduction at gauges No. 1 5. But the reductions of the amplitude in second and third harmonics are much less evident. And even on the contrary, in post-fluidized stage of Test B-4 $\left(U_{r}=14.90\right)$ and Test C-4 $\left(U_{r}=34.74\right)$, the second-harmonic amplitudes are found to be slightly increased. This indicates that the major cause of the wave decay of the waves in RF tests is due to the fluidization response of the bed soils. Meanwhile primary reductions of the amplitude happen in fundamental harmonic. In general, in post-fluidized stage the trends of the spatial distribution are similar to those in pre-fluidized stage.

\section{CONCLUSIONS}

In this study, the HHT for nonlinear and nonstationary data was applied to analyze the experimental wave records in detail. The EEMD with a post-processing method of MSEMD was used to decompose the surface waves into a set of modified IMF without any clear mode mixing. For UF tests, the decomposed IMF results have shown that amplitudes of the fundamental (first) harmonic components modulate less with time in the vicinity of the S.O. than those of the second and third harmonics. This indicates that higher harmonics are more susceptible to interactions between waves and the S.O.

For RF tests, significant decay of harmonic amplitudes is clearly observed during the transient development stage of the fluidized response in the sandy bed. On the other hand, in post-fluidized stage, the amplitude of fundamental and higher harmonics increases gradually due to slightly decreasing excess pore pressures. Furthermore, in transient stage the frequency ranges of higher harmonics become wider than those in pre- and post-fluidized stages. This suggests that in addition to the effects of S.O., the occurrence of bed soil's fluidization could further cause energy transferring among higher harmonics.

From the results of spatial distribution of mean amplitude of the wave spectra of each IMF, in the UF tests no clear dissipation in the fundamental harmonic can be found. However, as waves propagate upon an unfluidized sandy bed, the higher harmonics have slight growth in amplitude on leeward side of the S.O. than that above the S.O. Comparisons between UF tests and RF tests in pre-fluidized stage, there are obvious influences of the values of relatively higher $U_{r}$, on the spatial evolutions of amplitude around the S.O. The RF test with lower $U_{r}$ reveals similar trend to that of the UF test. Furthermore, in post-fluidized stage, fundamental-harmonic amplitudes are significantly reduced at all the 5 sites. But similar amplitude reductions can not be clearly identified in the second and third harmonics. On the contrary, the second-harmonic amplitudes have been found to slightly grow up with increasing the $U_{r}$.

\section{ACKNOWLEDGMENTS}

This study is financially supported by the National Science Council, Taiwan, Republic of China under project numbers of NSC 97-2221-E-019-042.

\section{REFERENCES}

1. Chen, Y.-L., Tzang, S.-Y., and Ou, S.-H., "Application of the EEMD method to investigate pore pressure build-ups in a wave-fluidized sandbed," Proceedings of the 31st International Conference on Coastal Engineering (ICCE 2008), Vol. 2, pp. 1614-1624 (2008).

2. Chen, Y.-L., Tzang, S.-Y., and Ou, S.-H., “Application of an EEMD post processing method using MSEMD to analyze wave-induced pore pressures in a fluidized bed," Ocean Engineering (2010). (Submitted)

3. Deering, R. and Kaiser, J. F., "The use of a masking signal to improve empirical mode decomposition," Proceedings of IEEE International Conference on Acoustics, Speech, and Signal Processing (ICASSP 2005), Vol. 4, pp. 485-488 (2005).

4. Huang, N. E., Shen, Z., Long, S. R., Wu, M. C., Shih, H. H., Zheng, Q., Yen, N. C., Tung, C. C., and Liu, H. H., "The empirical mode decomposition and the Hilbert spectrum for nonlinear and non-stationary time series analysis," Proceedings of the Royal Society A: Mathematical, Physical and Engineering Sciences, Vol. 454, pp. 903-995 (1998).

5. Losada, I. J., Patterson, M. D., and Losada, M. A., "Harmonic generation past a submerged porous step," Coastal Engineering, Vol. 31, No. 1-4, pp. 281-304 (1997)

6. Mansard, E. P. D. and Funke, E. R., "The measurement of incident and reflected spectra using a least squares method," Proceedings of the 17th International Conference on Coastal Engineering, Vol. 1, pp. 154-172 (1980).

7. Massel, S. R., "Harmonic generation by waves propagating over a submerged step," Coastal Engineering, Vol. 7, No. 4, pp. 357-380 (1983).

8. Ohyama, T. and Nadaoka, K., "Modeling the transformation of nonlinear 
waves passing over a submerged dike," Proceedings of the 23rd International Conference on Coastal Engineering, Vol. 1, pp. 526-539 (1992).

9. Tzang, S.-Y. and Ou, S.-H., "Laboratory flume studies on monochromatic wave-fine sandy bed interactions: Part 1. Soil fluidization," Coastal Engineering, Vol. 53, No. 11, pp. 965-982 (2006).

10. Tzang, S.-Y., Ou, S.-H., Yang, Y.-C., and Chen, Y.-L., "A flume study on regular wave transformation and bed scouring near a rectangular sub- merged obstacle upon a fluidized bed," 2008 Taiwan-Polish Joint Seminar on Coastal Protection, Nov. 6-7, Tainan, Taiwan, pp. B35-B48 (2008).

11. Wu, Z. and Huang, N. E., "Ensemble empirical mode decomposition: A noise-assisted data analysis method," Advances in Adaptive Data Analysis, Vol. 1, No. 1, pp. 1-41 (2009). 